\title{
Trivium
}

Revue franco-allemande de sciences humaines et sociales - Deutsch-französische Zeitschrift für Geistesund Sozialwissenschaften

29 | 2019

Regards croisés sur le prophète de l'Islam

\section{Der Prophet des Islams im Blickwechsel}

\section{Einleitung}

\section{Francesco Chiabotti et Ruggero Vimercati Sanseverino}

Traducteur : Bettina Engels

\section{OpenEdition}

\section{Journals}

Édition électronique

URL : http://journals.openedition.org/trivium/6393

DOI : $10.4000 /$ trivium.6393

ISBN : 1963-1820

ISSN : 1963-1820

\section{Éditeur}

Les éditions de la Maison des sciences de l'Homme

Référence électronique

Francesco Chiabotti und Ruggero Vimercati Sanseverino, "Der Prophet des Islams im Blickwechsel», Trivium [Online], 29 | 2019, online erschienen am 17 Oktober 2019, abgerufen am 08 September 2020. URL : http://journals.openedition.org/trivium/6393; DOI : https://doi.org/10.4000/trivium.6393

Ce document a été généré automatiquement le 8 septembre 2020

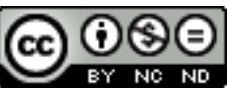

Les contenus des la revue Trivium sont mis à disposition selon les termes de la Licence Creative Commons Attribution - Pas d'Utilisation Commerciale - Pas de Modification 4.0 International. 


\title{
Der Prophet des Islams im Blickwechsel
}

\section{Einleitung}

\author{
Francesco Chiabotti et Ruggero Vimercati Sanseverino \\ Traduction : Bettina Engels
}

Die Idee, ein Heft über den Propheten des Islams zu machen, entstand im Laufe des ersten Jahres des von der Agence nationale de la recherche und der Deutschen Forschungsgemeinschaft geförderten deutsch-französischen Projekts "PROPHET Muhammad im Spiegel seiner Gemeinschaft im frühmodernen und modernen Islam «. ${ }^{1}$ Dieses großangelegte Forschungsprogramm, an dem viele Wissenschaftler ganz unterschiedlicher Spezialisierungen (sowohl geografisch gesehen wie im Hinblick auf ihre wissenschaftlichen Fachgebiete) beteiligt waren, hatte zum Ziel, die jeweiligen Eigenarten der französischen bzw. deutschen Islamforschung in Beziehung zu setzen. ${ }^{2}$ Beide Länder blicken auf eine reiche Tradition der historischen und literarischen Islamforschung zurück. Von Anbeginn standen in den sogenannten »orientalistischen« Fächern - mal fruchtbare, mal konfliktreiche - Begegnungen im Mittelpunkt bei der "Herausbildung dieser neuen Disziplin, die man als europäisch bezeichnen darf «, wie es Michel Espagne formuliert hat. ${ }^{3}$ Die Notwendigkeit der Zusammenarbeit gab den Anstoß zur Einrichtung einer Forschungsgruppe, in der die Rezeption der prophetischen Figur des Islams in Vormoderne, Neuzeit und Gegenwart aus ganz unterschiedlichen Perspektiven untersucht wurde. Das vorliegende Themenheft der Zeitschrift Trivium soll insgesamt einen Eindruck vom Reichtum der beiden Forschungstraditionen vermitteln, insbesondere aber vom Reichtum jener Forschung, die sich speziell der Figur des Propheten des Islams widmet.

Während das Projekt PROPHET chronologisch strukturiert war - und zunächst die vormoderne, dann die moderne Epoche untersuchte -, ist dieses Heft eher thematisch angelegt: Es konzentriert sich auf die Prozesse, die im Laufe der Zeit - und zwar seit den Ursprüngen des Islams - erst zu einer Sakralisierung, dann zu einer neuerlichen Infragestellung dieser Darstellung des Propheten geführt haben. Was der Leser also nicht finden wird, ist eine Debatte um die Geschichtlichkeit der Gestalt Muhammads. 
Schon zu Beginn des Projekts verständigten sich die Teilnehmer darauf, diese zwar vielversprechenden, aber schwierigen Forschungsfragen auszuklammern. ${ }^{4}$ Es ging uns vielmehr darum, zu zeigen, wie jede Epoche ihre eigene »Repräsentation« des Propheten entwickelte und wie auf diese Weise die "Gegenwart" des Propheten in seiner Gemeinschaft Wirklichkeit wurde. Eine erste Gruppe von Artikeln hinterfragt in diesem Heft deshalb das Bild des Propheten in älteren Textkorpora. Die Ausprägung dieses Bildes geht Hand in Hand mit der Festsetzung von Regeln und Normen, die der Übermittlung und Etablierung ebenjener Korpora zugrunde liegen. Der erste Artikel, »Mündliche Thora und Ḥadīth im Islam. Überlieferung, Schreibverbot, Redaktion«, von Gregor Schoeler ${ }^{5}$ war der erste einer langen Reihe von Beiträgen des Autors, die sich mit dem Verhältnis von Mündlichkeit und Schriftlichkeit im frühen Islam beschäftigen. ${ }^{6}$ Chronologisch gesehen beziehen sich diese Debatten sicherlich auf die Zeit nach der ursprünglichen Verkündung des Propheten und der Niederschrift des Korans. Ihre "Vorrangstellung" besitzen sie allerdings, weil sie bei der Etablierung und Übermittlung der historischen und hagiographischen Materialien über die Entstehung des Islams eine so entscheidende Rolle gespielt haben. In seiner Erörterung der Thesen Goldzihers (1850-1921) bringt Schoeler das Verbot des Übergangs zur Schriftlichkeit (das sich ja nicht durchgesetzt hat) in Zusammenhang mit der ganz ähnlichen Auseinandersetzung, die zu Zeiten der Niederschrift von Mischna und Talmud innerhalb des Judentums stattfand - zweier Korpora, die vor ihrer Verschriftlichung ebenfalls mündlich überliefert worden waren. Die Parallele zum Islam ist offensichtlich, denn auch diese neue Religion zeichnet sich dadurch aus, dass eine signifikante chronologische Lücke zwischen der schriftlichen Weitergabe des Korans und dem Verfassen der ersten Sammlungen prophetischer Überlieferungen besteht. Im hier vorliegenden Artikel bemüht sich der Autor um eine differenzierte Antwort auf die Frage nach der verzögerten schriftlichen Fixierung des Wissens in der Anfangszeit des Islams. Vor dem Hintergrund einer Analyse der prosopographischen Literatur und der Überlieferungsketten der Hadithe schlägt der Autor vor, dass mündliche und schriftliche Überlieferung in Wirklichkeit immer nebeneinander existiert haben. Die Rekonstruktion dieser Debatte erlaubt es uns, für eine sehr frühe Zeit den Status des prophetischen Wortes in der sich bildenden Gemeinschaft zu erfassen, ebenso wie das Zusammenspiel zwischen den unterschiedlichen Überlieferungsformen, mit denen sich die ersten Gelehrten auseinandersetzen mussten, um ihre eigene Methodologie für die Weitergabe des Wissens zu entwickeln. Man muss also diese manchmal etwas weitgespannte Dialektik im Hinterkopf behalten, um zu verstehen, woher das Korpus an Überlieferungen über den Propheten stammt, das in den Artikeln von Denis Gril ${ }^{7}$ und Samuela Pagani ${ }^{8}$ untersucht wird. Letztere widmet ihren Beitrag einer besonderen Episode im Leben des Propheten, nämlich der "Geschichte der Wahl«, in der »eine Stimme ohne Namen oder der Herr selbst den Propheten Muhammad dazu auf[fordert], sich zu entscheiden, ob er als Prophet ein König oder ein Diener sein wolle«. Wie die Autorin bemerkt, haben wir es hier mit zwei gegensätzlichen Typologien des Prophetentums zu tun: zum einen mit dem Paradigma des heiligen Königtums (und auch hier sind die Parallelen zur Bibel beachtenswert) und zum anderen mit dem der Unterwerfung unter Gott. Die Autorin fasst die Rolle, die diese Wahl für die ursprüngliche Gemeinschaft gespielt haben mag, folgendermaßen zusammen:

"Die Unterscheidung oder vielmehr der Gegensatz zwischen König und Diener wird in der Tradition weiterentwickelt und nimmt wichtige politische und theologische Konnotationen an, die zugleich das Wesen und die Funktion des Propheten betreffen. Die ১Erzählung von der Wahl inszeniert die Spannung zwischen diesen 
beiden Bestandteilen seines Bilds in Form einer Versuchungsgeschichte. Ganz wie die Erzählung von Jesu Versuchung in der Wüste spiegelt sie eine Debatte über das Wesen und die Funktion des Begründers, die bis in die erste Phase der Gemeinschaft zurückreicht, sich aber im Laufe ihrer Geschichte immer weiter entwickelt hat. ${ }^{9}$

Diese Dichotomie, von der auch die Figur des Propheten durchdrungen ist, hat im Laufe der gesamten Geschichte islamischer Gesellschaften zu einer Spannung zwischen dem Politischen und dem Religiösen geführt, zu ihrer Trennung oder aber, ganz im Gegenteil: zu ihrer Untrennbarkeit.

Während Samuela Paganis Artikel die Schwierigkeiten und nicht ausbuchstabierten Implikationen einer einzelnen alten Geschichte, eines einzigen alten Topos entfaltet, untersucht Denis Grils Text einen in der universitären Forschung weitgehend vernachlässigten Aspekt der muslimischen Darstellung des Propheten, nämlich die sakrale Bedeutung seines Körpers. Indem er zeigt, wie das muslimische Denken die Offenbarung des göttlichen Wortes mit einer Sakralisierung der Person und des Körpers seines Empfängers in Beziehung setzt, unterstreicht der Autor die theologische Grundlage der Verehrung prophetischer Reliquien, macht aber auch die Bindung der islamischen Überlieferung an die körperliche Beschreibung des Propheten verständlich. Die Person des Propheten erscheint in spiritueller wie physischer Hinsicht als Ort der Begegnung zwischen dem Transzendenten und dem Immanenten, und damit als dasjenige, durch das Raum, Zeit, Objekte und Personen sakralisiert werden. Auf diese Weise bringt die subtile Lektüre dieser Geschichten ein leibhaftiges Interesse ans Licht, das in der ersten Gemeinschaft groß gewesen sein muss, auch wenn die puritanischen Tendenzen des vormodernen und modernen Islams versuchten und bis heute versuchen, diese körperliche, ja wundertätige Gegenwart des Propheten im Schoße seiner Gemeinschaft auszuradieren. Es sei daher vermerkt, dass die ersten Abhandlungen über die Verehrung der Reliquien des Propheten geschrieben wurden, um sich gegen die Angriffe der Anhänger eines "puritanischen« Islams zu rechtfertigen, wie sie in den von Riexinger untersuchten Schriften des Muhammad 'Abd al-Wahhāb exemplarisch zum Ausdruck kommen.

4 Ausgehend von innovativen Fragestellungen ermöglicht diese neuartige Betrachtungsweise den Autoren, aus den kanonischen Hadith-Sammlungen eine Reihe von Fragen herauszudestillieren (Paradigma des Königtums in der Spätantike, zentrale Bedeutung des Körpers für das religiöse Charisma), deren Begrifflichkeit sich stark von derjenigen unterscheidet, die im überlieferten Korpus zur Sprache kommt. Damit wird die Frage nach der Begründung eines verbindlichen Modells und Vorbilds gestellt. Die ältesten islamischen Quellen erscheinen vor diesem Hintergrund als der Ausdruck des kollektiven Gedächtnisses und dessen, was es vom Propheten bewahren, oder gar generieren wollte. Es ging den Quellen dabei gleichermaßen um eine ideale Rekonstruktion des Prophetenbilds wie darum, Antworten auf die Herausforderungen ihrer jeweiligen Zeit zu finden: In den ersten Jahrhunderten des Islams musste ein Bild des Propheten als Heilsbringer geschaffen und zum Ausdruck gebracht werden, um die Rolle des Islams für die menschliche Heilsgeschichte zu bestimmen. Da die früheste Rezeption der Gestalt des Propheten im Kontext einer von eschatologischer Erwartung und Erlösungshoffnung geprägten Spätantike stattfand, wird der Messianismus hier ebenso behandelt wie soteriologische Motive.

5 Den zweiten Teil dieser Ausgabe bilden drei Artikel, die der Entwicklung der theologischen Debatte um die Figur des Propheten im vormodernen und modernen Islam nachgehen. 
Auch der Schweizer Orientalist Fritz Meier (1912-1998), ${ }^{10}$ dessen Werk weitgehend dem Studium der islamischen Mystik gewidmet war, leistete einen bemerkenswerten Beitrag zur Erforschung der theologischen Repräsentationen des Propheten des Islams. In dem von uns ausgewählten Artikel analysiert er im Detail eine Problematik der Prophetologie, an der sich die Geister im Laufe der Jahrhunderte besonders geschieden haben. Eine Reihe von Glaubensrichtungen scheint an der Post-mortem-Gegenwart des Propheten festzuhalten. Zur Erklärung, Stützung oder Widerlegung dieser These wurden über die Jahrhunderte diverse theologische Antworten gegeben, deren Nähe zum christlichen Auferstehungsglauben auffällig und, für manch einen, verstörend ist. Meiers Beitrag gliedert sich in zwei Teile: Ein erster Teil widmet sich der historischen Rekonstruktion dieser Debatte, in einem zweiten Teil werden die Thesen Suyūțīs (14451505), eines der großen Gelehrten des 15. Jahrhunderts, präsentiert. Diese Fragen spielen innerhalb der islamischen Gemeinschaft eine große Rolle. Abschließend erläutert Meier, in welchem Sinne die Debatte für die theologische Definition des Wahhabismus einerseits und des neuzeitlichen Sufismus andererseits von entscheidender Bedeutung sind. Damit nimmt er die beiden folgenden Artikel schon in gewissem Sinne vorweg. Der erste von ihnen ist eine neue Untersuchung Martin Riexingers, ${ }^{11}$ der eine kritische Lesart der MuHtașar sirat ar-rasūl (Das Leben des Propheten) vornimmt, einer vom Gründer der wahhabitischen Bewegung, Muhammad b. 'Abd al-Wahhab (1703-1792), verfassten Prophetenbiographie. Riexinger beleuchtet die textuellen Verfahren, die der Autor mit zweierlei Absicht anwendet: einerseits um die historische Gestalt des Propheten jedes übermenschlichen Charakters zu entledigen und andererseits, um im Zuge einer pseudohistorischen Umschrift (Riexinger weist dem Text zahlreiche Anachronismen nach) sein eigenes Handeln und seine eigene Ideologie zu legitimieren. Letzteres Anliegen versucht Ibn 'Abd al-Wahhāb zu erreichen, indem er Parallelen zwischen seinem eigenen politischen Handeln und dem des Propheten suggeriert.-Auf diese Weise kann Riexinger triftig zeigen, wie groß der Bruch des Wahhabismus mit dem traditionellen sunnitischen Denken ist.

Riexingers Artikel setzt beim Leser eine gründliche Kenntnis der wichtigsten Ereignisse im Leben des Propheten und der ersten Gemeinschaft voraus. Dieses Wissen ist jedoch nicht unbedingt notwendig, um einen wesentlichen Punkt zu verstehen, den der Autor aus der von Muhammad b. 'Abd al-Wahhāb (re)kostruierten Propheten-Biographie herausarbeitet. Anhand zahlreicher Beispiele zeigt Riexinger, in welch hohem Maße sich die politische Theologie der wahhabitischen Bewegung und ihr Geschichtsbild wechselseitig bedingen. Der Zusammenhang wäre vielleicht nicht so offensichtlich, wenn der Autor die Zerlegung und anschließende Neuzusammensetzung der Prophetenfigur bei Ibn 'Abd al-Wahhāb nicht mit solcher Klarheit herausgearbeitet hätte. Die ersten Autoren dieses im 9. Jahrhundert entstandenen literarischen Genres (der sira) hatten die Gestalt des Propheten als das ultimative Ziel der Schöpfung in eine weit ausgreifende Universalgeschichte eingebettet. Die MuHtașar von Muhammad b. 'Abd al-Wahhāb kommt zwar auf diese ursprüngliche Idee zurück, die prophetische Mission ist darin aber nur eine Episode in einem langen Kampf zwischen Gut und Böse. Ein ganzes Geschichtsbild steht hier also auf dem Prüfstein. Das (zur Debatte stehende) Dogma,$^{12}$ dem zufolge es nach Muhammad keinen neuen Propheten geben wird, führt die Menschheit in eine neue Phase ein, in der Gott die Menschen nicht mehr (direkt) für ihren Unglauben »bestraft«. Das unmittelbare Eingreifen Gottes in die Menschheitsgeschichte, um Gemeinschaften zu bestrafen, die nicht an seine Gesandten glauben, wird nun ersetzt durch das Wirken des Menschen, der die Botschaft des 
Monotheismus weiterträgt. So zumindest das Bild der wahhabitischen Bewegung von ihrer eigenen historischen Rolle. Die religiöse Überlieferung, die eine Heilsgeschichte ist, ist unter der Feder Muhammad b. 'Abd al-Wahhābs zu einer Geschichte der menschheitlichen Verdammnis geworden, die Riexinger $\mathrm{zu}$ Recht eine "Unheilsgeschichte« nennt.

7 Das hat unterschiedliche Folgen. Der Kampf gegen das Sakrale und die Verehrung der Heiligen wird durch diese überraschend moderne Darstellung einer entzauberten Welt verständlich. Im Gegensatz dazu gehen die klassischen und vormodernen Formulierungen des Sufismus und des Shiismus angesichts göttlicher Immanenz von einer permanenten Heilsbedeutsamkeit der Geschichte aus. Sie wird im sunnitischen Sufismus durch die Rolle der Heiligen zum Ausdruck gebracht und im Shiismus durch das Erbe der Imame, von denen der letzte jeweils immer noch lebt. ${ }^{13}$ Aus der Theologie Muhammad b. 'Abd al-Wahhābs speist sich also ein Geschichtsbild, das selbst aus dem Koran alles auszuschließen trachtet, was in Widerspruch zu seinem militärischen Programm geraten könnte. Hatte Mose im Koran seinem Volk noch vergeben können, dass es dem Götzendienst verfallen war (Geschichte vom Goldenen Kalb), so duldet Muhammad b. 'Abd al-Wahhāb nicht mehr die geringste Abweichung von dieser neuen Idee göttlicher Transzendenz. Vor diesem Hintergrund wird auch einsichtig, warum es falsch ist, diese Bewegung als »ultra-traditionalistisch« zu charakterisieren. Riexingers Artikel lässt keinen Zweifel daran, dass sich der Wahhabismus von Positionen abkehrt, die für die traditionelle sunnitische Identität maßgeblich waren.

8 Der Artikel von Michel Chodkiewicz ${ }^{14}$ analysiert einen weiteren Aspekt der für die Moderne charakteristischen Debatten um den Propheten. Er ist Teil einer historiographischen Untersuchung dessen, was die angelsächsische Forschung "Neosufismus « nennt, einer im 18. und 19. Jahrhundert entstehenden und reformierten Konfiguration des Sufismus, der die Rückkehr zum Vorbild des Propheten propagiert. ${ }^{15}$ Der Autor möchte zeigen, dass gewisse Aspekte dieser Strömung im mystischen Schrifttum keineswegs neu sind, sondern dass die Ansätze einer solchen Fokussierung auf den Propheten in der spirituellen Verwirklichung wesentlich älter sind, als man meinen könnte. Nun ist aber noch einmal darauf hinzuweisen, dass hier jenseits der historiographischen Debatte, wie sie innerhalb eines Fachpublikums geführt wird, eine bestimmte Vorstellung von der "Geschichtlichkeit« des Propheten wesentlich ist. Der historische Abstand zur Epoche des Propheten hat nicht nur zu einer Idealisierung dieses für immer verlorenen goldenen Zeitalters geführt (Anspruch der salafiyya ist die Rückkehr zur Praxis der »frommen Vorfahren«). In den spirituellen Strömungen sind neue Varianten einer Vergegenwärtigung des Propheten zu beobachten: neue Rituale, neue Techniken, neue Formen der Andacht, und auch das, was Meier einer Analyse der Abhandlung des Suyūṭī entnimmt, nämlich die Verbreitung von Wach-Visionen der Begegnung mit einem »lebendigen« Propheten, der sich nicht aus der Geschichte zurückgezogen hat, sondern vielmehr an ihr teilnimmt - sicherlich auf einer »esoterischen« Ebene, die einer Elite von Heiligen vorbehalten bleibt, was die Anhänger dieser neuen Prophetologie, die den unmittelbaren Zugang zur Figur des Propheten predigt, allerdings nicht daran hindert, politisch aktiv zu werden und damit die Gegenwart neu zu besetzen.

9 Die vorliegende Einführung versteht sich als Leitfaden für Nicht-Fachleute. Wir wollen nicht schließen, ohne der Trivium-Redaktion zuvor für den exzellenten Austausch zu danken, in dem wir vom ersten Moment an mit ihr gestanden haben. Wir möchten auch 
den Übersetzern und Redakteuren, insbesondere Katrin Heydenreich und Bernd Schwibs, für ihre Geduld und aufmerksame Bearbeitung der Texte danken.

Dieses Konvolut von Artikeln, das hier Lesern beider Sprachen zur Verfügung gestellt wird, reagiert auf einen großen Wissensdurst im Hinblick auf die Gründerfigur des Islams. Die Auswahl der Artikel verdankt sich auch pädagogischen Überlegungen. In Anbetracht der Tatsache, dass es eine Renaissance der Islamwissenschaften in Frankreich gibt (innerhalb von zwei Jahren wurden etwa zehn Stellen geschaffen) und dass auch in Deutschland mittlerweile Institute für Islamische Theologie eingerichtet worden sind, ist eine grenzüberschreitende Lektüre der Quellen sicherlich geeignet, Fragestellungen rund um den Islam in Europa zu bereichern und zusammenzuführen.

\section{BIBLIOGRAPHIE}

Amir-Moezzi, M. A. (1992): Le Guide divin dans le shi'isme originel. Aux sources de l'ésotérisme en Islam, Collection Islam Spirituel, Paris-Lagrasse: Verdier.

Brockopp, J. E. (2016): »Islamic Origins and Incidental Normativity «, Journal of the American Academy of Religion, 84 (1), S. 28-43.

Espagne, M. (2008): »Silvestre de Sacy et les orientalistes allemands«, Revue germanique internationale, 7 [online]; http://journals.openedition.org/rgi/398 (online verfügbar seit 15. Mai 2011, zuletzt abgerufen am 21. Mai 2019); https://doi.org/10.4000/rgi.683.

Espagne, M. / Lafi, N. / Raubault-Feuerhahn, P. (Hg.) (2014): Silvestre de Sacy. Le projet européen d'une science orientaliste, Paris: Éditions du Cerf.

Gril, D. (2006): »Le corps du Prophète«, Revue des mondes musulmans et de la Méditerranée 113/114: Le corps sacré en Orient musulman, hg. von B. Heyberger / C. Mayeur-Jaouen, S. 37-57.

Groß, M. / Ohlig, K.-H. (Hg.) (2017): Die Entstehung einer Weltreligion IV. Mohammed - Geschichte oder Mythos?, Berlin / Tübingen: Schiler.

Hofheinz, A. (1996): »Illumination and Enlightenment Revisited, or: Pietism and the Roots of Islamic Modernity«, Vortrag an der Universität Bergen, 19. Sept. 1996 [online verfügbar: https:// folk.uio.no/albrech/Hofheinz_IllumEnlightenment.pdf].

Pagani, S. (2017): ")Roi ou serviteur<? La tentation du Prophète, ou le choix d'un modèle«, Archives des sciences sociales des religions, 178: Le Prophète en islam: L'instauration d'un modèle et formes de dévotion, hg. von Amri, N. / Chih, R. / Gril, D., S. 43-67.

Riexinger, M. (2015): » "Der Islam begann als Fremder, und als Fremder wird er wiederkehren«. Muhammad b. 'Abd al-Wahhabs Prophetenbiographie MuHtașar sīrat ar-rasūl als Programm und Propaganda«, Die Welt des Islams, 55, S. 1-61.

Sangaré, Y. (2018): Le scellement de la prophétie en islam, Paris: Geuthner.

Schoeler, G. (1989): »Mündliche Thora und Ḥadīth im Islam. Überlieferung, Schreibverbot, Redaktion«, Der Islam, 66, S. 213-251. 
Schoeler, G. (Hg.) (2002): Écrire et transmettre dans les débuts de l'islam, Paris: Presses universitaires de France.

\section{NOTES}

1. https://prophet.hypotheses.org, von Rachida Chih (CETOBAC - CNRS) und Stefan Reichmuth (Universität Bochum) geleitetes Projekt.

2. Das Projekt hat drei Schwerpunkte:

1. Repräsentationen des Propheten: Figurationen und Kontroversen;

2. Erben des Propheten: Legitimation und Begründung religiöser Autorität und politischer Macht; 3. prophetische Frömmigkeit zwischen individueller und kollektiver Erfahrung.

3. Michel Espagne (2008): "Silvestre de Sacy et les orientalistes allemands«, Revue germanique internationale, 7 [online]; http://journals.openedition.org/rgi/398 (online verfügbar seit 15. Mai 2011, zuletzt abgerufen am 21. Mai 2019); https://doi.org/10.4000/rgi.683. Siehe auch Silvestre de Sacy. Le projet européen d'une science orientaliste, unter der Leitung von Michel Espagne, Nora Lafi und Pascale Raubault-Feuerhahn, Paris: Éditions du Cerf, 2014.

4. Auf diesem Gebiet lassen sich die wichtigsten wissenschaftlichen Fortschritte fast ausnahmslos auf die Arbeit deutscher oder deutschsprachiger Forscher wie Harald Motzki, Gregor Schoeler, Andreas Gorke und Nicolai Sinai zurückführen. Erwähnt sei auch die Forschungsgruppe »Inârah" (Institut zur Erforschung der frühen Islamgeschichte und des Korans; http://inarah.net), die seit Jahren Sammelbände auf Deutsch herausgibt, in denen die sogenannte »hyperkritische« Methode angewendet wird. Aufgrund der geringen Verbreitung des Deutschen als Wissenschaftssprache sind die Arbeiten dieser Forschungsgruppe im Ausland nach wie vor wenig bekannt. Doch seitdem vor einigen Jahren die englische Übersetzung ihres ersten Bandes erschien, gerät sie nun allmählich auch in den Fokus der Aufmerksamkeit (und der Ängste) der angelsächsischen Forschung; vgl. die kritische Auseinandersetzung von Jonathan E. Brockopp in »Islamic Origins and Incidental Normativity «, Journal of the American Academy of Religion, 84 (1), 2016, S. 28-43. Der letzte Band konzentriert sich auf die Erscheinung Muhammads als historischer Figur. Mehrere Beiträge zu dem Band stellen kurzerhand die Realität seiner Existenz in Frage. Dieser Ansatz ist in der wissenschaftlichen Forschung hoch umstritten. Siehe Die Entstehung einer Weltreligion IV. Mohammed - Geschichte oder Mythos?, hg. von Markus Groß und Karl-Heinz Ohlig (Inârah - Schriften zur frühen Islamgeschichte und zum Koran, Bd. 8), Berlin / Tübingen: Schiler, 2017.

5. Gregor Schoeler (1989): »Mündliche Thora und Ḥadīth im Islam. Überlieferung, Schreibverbot, Redaktion«, Der Islam, 66, S. 213-251.

6. Auf Französisch ist vom selben Autor erhältlich: Écrire et transmettre dans les débuts de l'islam, Paris: Presses universitaires de France, 2002.

7. Denis Gril (2006): »Le corps du Prophète«, Revue des mondes musulmans et de la Méditerranée, 113/114: Le corps sacré en Orient musulman, hg. von B. Heyberger / C. Mayeur-Jaouen, S. 37-57.

8. Samuela Pagani (2017): ")Roi ou serviteur ? La tentation du Prophète, ou le choix d'un modèle«, Archives des sciences sociales des religions, 178: Le Prophète en islam: L'instauration d'un modèle et formes de dévotion, hg. von N. Amri / R. Chih / D. Gril, S. 43-67.

9. Pagani (2017), S. 43.

10. http://www.iranicaonline.org/articles/meier-fritz-1

11. Martin Riexinger (2015): ")Der Islam begann als Fremder, und als Fremder wird er wiederkehren`. Muhammad b. 'Abd al-Wahhabs Prophetenbiographie MuHtașar sīrat ar-rasūl als Programm und Propaganda«, Die Welt des Islams, 55, S. 1-61.

12. Vgl. das Buch von Yousef Sangaré (2018): Le scellement de la prophétie en islam, Pais: Geuthner. 
13. Vgl. Mohammed Ali Amir-Moezzi (1992): Le Guide divin dans le shi'isme originel. Aux sources de l'ésotérisme en Islam, Collection Islam Spirituel, Paris-Lagrasse: Verdier.

14. Michel Chodkiewicz (1994): "Le modèle prophétique de l'islam«, Al-Masāq - Journal of the Medieval Mediterranean 7, S. 201-226.

15. Siehe die Synthese durch Albrecht Hofheinz (1996): »llumination and Enlightenment Revisited, or: Pietism and the Roots of Islamic Modernity«, Vortrag an der Universität Bergen, 19. Sept. 1996 [online verfügbar: https://folk.uio.no/albrech/Hofheinz_IllumEnlightenment.pdf]. Siehe auch Catherine Mayeur-Jaouen: ">Aufklärung islamique`, ınéo-soufisme`, >réformisme musulman`, >islamismes` Renouveaux historiographiques de l'histoire de l'islam moderne et contemporain depuis les années 1960«, online: https://ahcesr.hypotheses.org/files/2016/04/ AHCESR-nov-2009.pdf.

INDEX

Mots-clés : islam, prophète, Muhammad

Schlüsselwörter : Islam, Prophet, Muhammad

\section{AUTEURS}

\section{FRANCESCO CHIABOTTI}

Francesco Chiabotti ist Dozent für Islamwissenschaften am Institut für orientalische Sprachen und Kulturen (INALCO) in Paris und Forscher am CETOBaC. Weitere Informationen finden Sie hier.

\section{RUGGERO VIMERCATI SANSEVERINO}

Ruggero Vimercati Sanseverino ist Juniorprofessor an der Universität Tübingen. Weitere Informationen finden Sie hier. 